\title{
Malposition of a Nasogastric Feeding Tube Into the Right Pleural Cavity of a Nasopharyngeal Carcinoma Patient After Radiotherapy and Chemotherapy: A Case Report
}

\author{
Meng Zhang \\ West China Hospital, Sichuan University https://orcid.org/0000-0003-0061-9113 \\ Hong Zhu ( zh-jxd@163.com) \\ West China Hospital, Sichuan University \\ Zheng Liu \\ West China Hospital, Sichuan University \\ Xuexue Deng \\ West China Hospital, Sichuan University
}

\section{Case report}

Keywords: Nasopharyngeal carcinoma, Malposition, Nasogastric feeding, Radiotherapy, Chemotherapy, Case report

Posted Date: November 15th, 2021

DOI: https://doi.org/10.21203/rs.3.rs-1022308/v1

License: (c) (i) This work is licensed under a Creative Commons Attribution 4.0 International License.

Read Full License 


\section{Abstract}

Background: Nasogastric feeding tube plays an important role in nutrition intake, drug administration, and stomach emptying for patients with severe dysphagia. However, inserting nasogastric tubes is not absolutely harmless. Inadvertent malposition into the trachea or the pleural cavity could result in severe pulmonary complications.

Case presentation: We present a case of a 67-year-old patient with a history of nasopharyngeal carcinoma and after the treatment of radiotherapy and chemotherapy. Nasogastric tubes have to be placed for enteral nutrition and avoiding aspiration owing to his severe dysphagia. Unfortunately, he experienced a malposition of nasogastric tube into the right pleura cavity after blind replacement by nurse, whereas the nurses and physicians did not recognize this fault, even the bedside chest radiography (X-ray) was performed twice after intubation. A week later, his condition deteriorated so rapidly that he had to undergo tracheotomy, and the tube was finally found in his trachea.

Conclusions: The Nasopharyngeal carcinoma patients after radiotherapy and chemotherapy should be fully evaluated before the nasogastric tube placement whether the blind insertion is suitable or not. Meanwhile, we should not feed immediately unless we have a radiograph to verify the right position of NG tube. Furthermore, careful monitoring of both typical and untypical symptoms of malposition is essential during tube feeding.

\section{Background}

Nasopharyngeal carcinoma (NPC) is a squamous cell carcinoma arising from the nasopharyngeal mucosal lining. According to the International Agency for Research on Cancer, about 129,000 new cases of NPC were reported in 2018[1]. Comparing with other cancers, nasopharyngeal carcinoma is uncommon. Nevertheless, more than $70 \%$ of new cases occurred in east and southeast Asia. In China, with an age-standardized rate of 3.0 per 100, $000[1,2]$. Radiotherapy is the main treatment modality for non-metastatic disease. Although NPC has led to increased survival rates, it causes dysphagia, mucositis, and xerostomia[3, 4]. Among them, dysphagia is one of the most prevalent late adverse effects of radiotherapy in patients with NPC [5, 6], which will have a negative influence on the patient's quality of life.

Tube feeding is widely used in acute and chronic care settings, especially for the patients with dysphagia. In general, Nasogastric (NG) tube insertion is carried out for various diagnostic and treatment purposes. For example, a catheter is inserted into the gastrointestinal though nasal cavity for supplying nutrition and/or giving medications to patients who were unable to eat via mouth. Previous study has showed that approximately 10 million NG feeding tubes are used per year in Europe, 1 million per year in the United Kingdom[7]. It requires the operator's advanced skills to safely perform insertion. In general, the NG tubes are intubated by nurses at the bedside. Actually, it is the nurse's responsibility to insert the NG tube, assure the safe maintenance, and administer medication or nutrients to the patients in China. 
There may be improper placement during the operation, such as the nasogastric tube strayed into the airway, or the position changes due to various reasons during the indwelling period[ 8,9$]$. The error rate of tube placement in adult patients is as high as $1.3 \%-50 \%[10]$. Another study also showed $59 \%$ of feeding tubes were placed incorrectly in the neonatal intensive care unit[11]. Misplacement of feeding tubes could induce severe pulmonary complications, including aspiration, pneumonia, pneumothorax, emphysema, pulmonary hemorrhage, atelectasis, and mortality $[12,13]$. According to the National Security Agency (NPSA) reported[10], 21 patients died and 79 patients were injured due to the wrong position of the gastric tube between 2005 and 2010 in the United Kingdom. In the United States, about 3600 8400 cases had lung injuries and 1200 3600 deaths occurred annually because of the incorrect placement of nasogastric tubes [14].

Therefore, inserting the gastric tube correctly and judging the position of the gastric tube accurately are vital for patients. Here, we present a case about a misplacement of the nasogastric tube in an old man with a history of nasopharyngeal carcinoma, causing a series of disastrous events.

\section{Case Presentation}

A 67-year-old man was admitted to the emergency department (ED) of our hospital on March 6th, 2021 because of cough, sputum, and mental abnormalities (e.g. agitation, dysphoria, sleep disturbances, hallucination) for 10 days. His medical history included chronic obstructive pulmonary disease (COPD) for more than 30 years, and nasopharyngeal carcinoma for 20 years. After radiotherapy combined the chemotherapy for nasopharyngeal carcinoma, the patient had hoarseness, dysphagia, restriction of mouth opening, and coughing while eating. Once he was admitted to ED, vital signs including body temperature, heart rate, respiratory rate, blood pressure, and pulse oxygen saturation were $36.5^{\circ} \mathrm{C}$, $80 / \mathrm{min}, 23 / \mathrm{min}, 90 / 59 \mathrm{mmHg}$, and $96 \%$, respectively. Besides, the chest radiography (X-ray) showed lowdensity nodules in the anterior mediastinum, emphysema and pulmonary bullae, interstitial changes in both lungs, and calcification of the aorta and right coronary artery (Figure 1). Therefore, his present diagnoses including type II respiratory failure and acute exacerbation of chronic obstructive pulmonary disease (AECOPD).

On March 9th, this patient was transferred to the respiratory department. As he could not eat orally, a no.4.67cm ( $\mathrm{CH} 14-110)$ of Flocare Nasogastric Feeding tube with guide wire was inserted by nurses at the bedside. During the insertion procedure, the patient had no complaints such as choking, dyspnea, nausea, etc. The operator aspirated stomach contents and auscultated epigastric to confirm tube position. Even though no contents were drawn out, gurgling was heard. About two hours later, $200 \mathrm{ml}$ nutrient solution was fed through NG tube, and cough was not observed during the feeding process. Tube feeding $200 \mathrm{ml}$ was performed three times a day in the follow-up nutrition supplement.

On March 15th, the oxygen saturation of this patient decreased to $85 \%$, and sputum was difficult to cough up. A nasal cannula oxygen (flow rate 1 2 L/min) was used immediately. His arterial blood gas (ABG) analysis showed $\mathrm{pH} 7.46$, oxygen partial pressure $\left(\mathrm{PaO}_{2}\right) 49.4 \mathrm{mmHg}$, and carbon dioxide partial 
pressure $\left(\mathrm{PaCO}_{2}\right) 41.0 \mathrm{mmHg}$. We changed the nasal cannula oxygen (flow rate $1 \sim 2 \mathrm{~L} / \mathrm{min}$ ) to mask oxygen (flow rate $6 \mathrm{~L} / \mathrm{min}$ ). As a result, oxygen saturation gradually increased to $96 \%$. In addition, his body temperature were examined with values of $38.8^{\circ} \mathrm{C}$. As the patient complained of acute pain in the upper edge of the right rib, a bedside chest radiography (X-ray) was performed and suggested that both lungs are scattered in patches, cords, nodules, and multiple inflammatory foci, and large areas of slightly higher density are seen in the lower lobe of the right lung (Figure 2). In the meantime, tramadol $100 \mathrm{mg}$ was injected intramuscularly for pain relief.

Unfortunately, his condition deteriorated rapidly, referring to the oxygen saturation dropping to $79 \%$ on March 16th. Repeated sputum aspiration, a large amount of thin bloody sputum was aspirated. Thus, gastrointestinal decompression was performed, and hemorrhagic fluid was drained for about $200 \mathrm{ml}$ from NG tube. Consequently, he received non-invasive mechanical ventilation, but the oxygen saturation still could not be maintained. $A B G$ analysis showed that $\mathrm{pH} 7.25, \mathrm{PaO}_{2} 53.2 \mathrm{mmHg}, \mathrm{PaCO}_{2} 100.8 \mathrm{mmHg}$. Because of his critical condition with severe pneumonia, respiratory failure, gastrointestinal bleeding, acid-base imbalance, and coagulation disorder, the anesthesiologist planned to perform endotracheal intubation at the bedside. However, because of the patient's face muscle contracture, it was difficult to open his mouth. Subsequently, we transferred him to the operating room for tracheotomy. During the tracheotomy procedure, the NG tube was found in his airway. Finally, he was transferred to Medical Intensive Care Unit (MICU) for further treatment.

In the MICU, the patient's condition worsened, e.g. he was under sedation, with invasive ventilator assisted ventilation in $A / C(V C)$ mode, and the electrocardiogram monitoring showed blood pressure 122/85 $\mathrm{mmHg}$, heart rate $101 / \mathrm{min}$, oxygen saturation $98-100 \%$. Dry and wet rales were heard in both lungs, arrhythmia was diagnosed. As his family applied to automatic discharge urgently on March 20, 2021, the prognosis of this patient was not traced further.

\section{Discussion}

Enteral tube feeding, especially the bedside NG tube feeding, is a crucial feeding method used in patients who are unable to meet their nutrition needs orally. However, it might be associated with side events complications and may worsen the prognosis of patients[15]. With the development of the position determining methods, the incidence of malposition has decreased to a certain extent. Nevertheless, during the blind bedside placement, inadvertent malposition into the lung, trachea or the pleural cavity still occurs frequently. We described the case of an elderly man who presented with malposition of nasogastric tube into the right pleural space. In this patient, NG tube placement failure was mainly caused by his anatomical issues, the methods to ensure NG tube position, the intubation method, or it may be related to the auxiliary means.

Anatomically, this patient suffered from nasopharyngeal carcinoma after radiotherapy and chemotherapy, movement and sensory disorders in throat muscle. Thus, he was at high risk of NG tube misplacement. This was in accordance with previous studies, among patients treated with radiotherapy, 
dysphagia occurs frequently by damage to neural and soft tissues[16], such as soft tissue fibrosis and neurological impairment[17]. In addition, Honghong $L$ et al. reported radiotherapy often alters the sensitivity of the swallowing structures in patients with nasopharyngeal carcinoma, which may result in a deficient cough reflex[18]. The cough reflex disappearance can cause misplacement of the tube in the trachea. We speculated this was the reason why this patient underwent NG tube malposition without symptoms such as cough during inserting and feeding process.

Since severe complications or death can be caused by misplaced NG tubes into the lung or tracheobronchial tract, it is of vital importance to the methods that confirming the position of NG tube. At present, various methods are employed to determine the placement of the NG tube, including radiography, respiratory distress, $\mathrm{pH}$ test, auscultation, carbon dioxide detection, auscultation, and enteral access devices[19]. Among these testing methods, radiography is regarded as the gold standard to distinguish between gastric and pulmonary placement[20-22]. Of non-radiographic methods, $\mathrm{pH}$ testing was the most popular method, and epigastric auscultation is least favored[23]. In our case, the nurse confirmed the position together with the doctor through suction of gastric contents and epigastric auscultation through injecting air into the gastric tube, which were commonly used. Besides, $40 \mathrm{ml}$ warm water was fed through NG tube, and the patient had no adverse reactions such as choking, signs of respiratory distress, etc. Nevertheless, the bedside radiography was not carried out immediately because of its deficiency, such as delayed enteral feeding administration and the risk of radiation exposure to patients [24-26]. Unfortunately, this patient encountered malposition of the NG tube. This adverse event indicated that the reliability of generally confirmation methods we chose with simple operation in clinical practice still needed to be validated. Previous research has proved that even if the NG tube is mal-positioned in the lung, or in the pleural cavity after perforation, the sound may still be heard. And a small amount of fluid aspirated may not be distinguished from tracheal secretion[27]. Therefore, as long as the patients at high risk of NG tube malposition, they should receive a radiograph to confirm the proper position of NG tube before initiating feedings[28]. However, it was worth noted that the radiologist performed bedside chest radiography for this patient twice because of severe chest pain, but they did not report the gastric tube was in the lungs or bronchi. Possible explanation was that the relationship of image quality and radiation dose was more complex in the routine radiography practice [29]. On the other hand, the silicone NG tube may not develop clearly because of the imprecise radiation dose or increased image noise during the radiography process [30]. Though radiographic examination is the most accurate method, this finding implied that the radiologist should choose the right radiation dose to ensure excellent image quality if we aimed to verify the position of NG tube.

Traditionally, the NG tube insertion is performed by nursing staff blindly, as we know, this requires skilled operational skills and rich practical experience. Besides, the anesthetic or instrument may be used to assist NG tube insertion. In this patient, the front end of the NG tube was smeared with tetracaine hydrochloride gel before intubation to ensure insert more smoothly. Actually, application of tetracaine hydrochloride gel could reduce the occurrence of uncomfortable reactions such as vomiting and coughing during intubation [31, 32]. Using it to perform surface anesthesia on patient's nasal cavity and throat mucosa that causes temporary loss of sensation, reduces the sensitivity of the superior laryngeal 
nerve, thus effectively easing the patient's nausea and vomit. However, for patients with impaired swallowing and muscle function, it will cover the adverse alert such as irritating cough that indicates the gastric tube enters the airway by mistake. Therefore, in our case, as this patient suffered from nasopharyngeal carcinoma after radiotherapy and chemotherapy, the tetracaine hydrochloride gel should not be used commonly.

Fortunately, misplacement of the gastric tube into the lung or trachea will be avoided under direct visualization using a video laryngoscope. Video laryngoscope-assisted nasogastric tube placement enables the operator to see if the tube has or has not entered the esophagus and to correct any nonesophageal placement, and this way helps advance the tube correctly into the esophagus[33]. Especially for patients with nasal contraindications who are influenced by physiological and pathological factors, traditional methods are not easy to succeed. A Meta-analysis compared the gastric tube insertion under video laryngoscope with the gastric tube insertion immediately, the results indicated insertion under video laryngoscope significantly reduce the incidences rate of stray trachea [34]. Tadashi's study showed that misplacement of the gastric tube in the trachea could been detected and corrected under the vision of video laryngoscope [35]. Hence, the operator could insert the NG tube with the assist of a video laryngoscope to avoid failure [36].

Most importantly, we should observe the patient's condition closely, and pay attention to his chief complaint of symptoms. For this patient, even though he had no choke after NG tube insertion, severe chest pain was reported repeatedly. This might be the vital signal of NG displacement into the lung or trachea. Unfortunately, our physicians and nurses ignored this crucial signal that indicated the NG tube of displacement.

\section{Conclusion}

Successful NG tube catheterization can reduce the suffering of the patients' prognosis of nasopharyngeal carcinoma. The lessons from our case suggested that high-risk patients with nasopharyngeal cancer should be fully evaluated before the NG tube placement whether the blind insertion is suitable or not. Meanwhile, we should not simply take it for granted that the tube is just in the right place, unless we have a radiograph to confirm the right position before initiating feeding.

Furthermore, in the face of special symptoms, especially typical symptoms of malposition, should be dealt with promptly. These are crucial methods to promote the success rate of intubation for such highrisk patients.

This study was conducted according to Case Report Guidelines (CARE).

\section{Abbreviations}

NPC

Nasopharyngeal carcinoma 
NG

Nasogastric

NPSA

National Security Agency

ED

emergency department

COPD

chronic obstructive pulmonary disease

X-ray

radiography

AECOPD

acute exacerbation of chronic obstructive pulmonary disease

$A B G$

arterial blood gas

$\mathrm{PaO}_{2}$

oxygen partial pressure

$\mathrm{PaCO}_{2}$

carbon dioxide partial pressure

MICU

Medical Intensive Care Unit.

\section{Declarations}

\section{Ethics approval and consent to participate}

Ethics approval was obtained from the Research Ethics Committee of West China Hospital of Sichuan University.

\section{Consent for publication}

Written informed consent was obtained from the patient's family for publication of this case report.

\section{Availability of data and materials}

The data supporting the conclusions of this article are included within the manuscript.

\section{Competing interests}

The authors declare that they have no competing interests.

\section{Funding}

Not applicable 


\section{Authors' contributions}

Meng Zhang and Hong Zhu designed the research; Zheng Liu and Xuexue Deng conducted the research; Zheng Liu, Hong Zhu and Xuexue Deng performed case analysis; Meng Zhang wrote the paper; Hong Zhu had responsibility for final content. All authors read and approved the final manuscript.

\section{Acknowledgements}

All coauthors have agreed to the submission and publication of this manuscript. The authors would like to thank the nursing staff in the Department of Respiratory Medicine of West China Hospital, Sichuan University.

\section{References}

1. Bray F, Ferlay J, Soerjomataram I, et al.Global cancer statistics 2018: GLOBOCAN estimates of incidence and mortality worldwide for 36 cancers in 185 countries. CA Cancer J Clin. 2018; 68(6);394-424. doi: 10.3322/caac.21492.

2. Ferlay J, Ervik M, Lam F, et al. Global Cancer Observatory: xancer today. Lyon, France: International Agency for Research on Cancer 2018. https://gco.iarc.fr/today (accessed Dec 28, 2018).

3. Arslan SA. Clinical Outcomes of Nasopharyngeal Carcinoma Patients Treated with Adaptive Helical Tomotherapy, A 5-year Experience. Niger J Clin Pract. 2020; 23(12);1683-1689. doi: 10.4103/njcp.njcp_647_19.

4. Li H, Li L, Huang X, Li Y, et al.Radiotherapy-induced dysphagia and its impact on quality of life in patients with nasopharyngeal carcinoma. Strahlenther Onkol. 2019; 195(6); 457-467. doi: 10.1007/s00066-018-01421-6.

5. Lertbutsayanukul C, Prayongrat A, Kannarunimit D,et al. A randomized phase III study between sequential versus simultaneous integrated boost intensity-modulated radiation therapy in nasopharyngeal carcinoma. Strahlenther Onkol. 2018;194(5);375-385. doi: 10.1007/s00066-0171251-5.

6. Sahai P, Mohanti BK, Sharma A, et al. Clinical outcome and morbidity in pediatric patients with nasopharyngeal cancer treated with chemoradiotherapy. Pediatr Blood Cancer. 2017; 64(2);259-266. doi: $10.1002 / p b c .26240$.

7. Torsy T, Saman R, Boeykens K, et al. Accuracy of the corrected nose-earlobe-xiphoid distance formula for determining nasogastric feeding tube insertion length in intensive care unit patients: $A$ prospective observational study. Int J Nurs Stud. 2020;110;103614. doi:

10.1016/j.ijnurstu.2020.103614.

8. Giantsou E, Gunning KJ. Blindly inserted nasogastric feeding tubes and thoracic complications in intensive care. Health.2010; 2(10);1135-1141. DOI: 10.4236/health.2010.210166

9. Anziliero F, Corrêa AP, Silva BA, et al. Nasoenteral tube: factors associated with delay between indication and use in emergency services. Rev Brasileira de Enfermagem. 2017;70(2);326-34. doi: 
10.1590/0034-7167-2016-0222.

10. National Patient Safety Agency $\mathbb{R}$ Reducing the harm caused by misplaced nasogastric feeding tubes in adults, children and infants [EB/OL] (2011.03.10)[2020-10-10].http://www.rls.psa.hs.k/resoureed? entryid45=129640\&p-4. 2011.

11. Quandt $D$, Schraner $T$, Ulrich Bucher $H$, et al. Malposition of feeding tubes in neonates: is it an issue? .J Pediatr Gastroenterol Nutr. 2009;48(5);608-11. DOI: 10.1097/MPG.0b013e31818c52a8

12. Sparks DA ,Chase DM ,Coughlin LM , et al. Pulmonary complications of 9931 narrow-bore nasoenteric tubes during blind placement: a critical review. JPEN J Parenter Enteral Nutr. 2011; 35(5),625-9 . doi: 10.1177/0148607111413898.

13. DeLegge MH. Enteral Access and Associated Complications. Gastroenterol Clin North Am. 2018; 47(1); 23-37. doi: 10.1016/j.gtc.2017.09.003.

14. Krenitsky J. Blind bedside placement of feeding tubes: treatment or threat? Pract Gastroenterol. 2011; 35(3);32-42.

15. Rowat A. Enteral tube feeding for dysphagic stroke patients .Br J Nurs. 2015;24(3);138-145. doi:10.12968/bjon.2015.24.3.138

16. Feng FY, Kim HM, Lyden TH, et al. Intensity-modulated chemoradiotherapy aiming to reduce dysphagia in patients with oropharyngeal cancer: clinical and functional results. J Clin Oncol. 2010; 28(16); 2732-2738. doi: 10.1200/JC0.2009.24.6199.

17. Schindler A, Denaro N, Russi EG, et al. Dysphagia in head and neck cancer patients treated with radiotherapy and systemic therapies: Literature review and consensus. Crit Rev Oncol Hematol. 2015;96(2);372-84. doi: 10.1016/j.critrevonc.2015.06.005.

18. Li H, Li L, Huang X, Li Y, et al. Radiotherapy-induced dysphagia and its impact on quality of life in patients with nasopharyngeal carcinoma.Strahlenther Onkol. 2019;195(6);457-467. doi: 10.1007/s00066-018-01421-6.

19. Metheny NA, Krieger MM, Healey F, et al. A review of guidelines to distinguish between gastric and pulmonary placement of nasogastric tubes. Heart Lung.2019; 48(3);226-235. doi: 10.1016/j.hrtlng.2019.01.003.

20. Godoy MC, Leitman BS, de Groot PM, et al. Chest radiography in the ICU: Part 1, Evaluation of airway, enteric, and pleural tubes . AJR Am J Roentgenol.2012; 198(3);563-71 . doi: 10.2214/AJR.10.7226.

21. Metheny NA, Meert KL. Monitoring feeding tube placement. Nutr Clin Pract. 2004; 19(5); 487-95. DOID10.1177/0115426504019005487

22. Elpern EH, Killeen K, Talla E, et al. Capnometry and air insufflation for assessing initial placement of gastric tubes. Capnometry and air insufflation for assessing initial placement of gastric tubes. Am J Crit Care. 2007;16(6); 544-9. PMID: 17962498.

23. Initial and ongoing verification of feeding tube placement in adults (applies to blind insertions and placements with an electromagnetic device). Crit Care Nurse. 2016;36(2);e8-e13. doi: $10.4037 / \operatorname{ccn} 2016141$. 
24. Soylu H, Wiseman NE, El-Sayed Y, et al. Radiographic confirmation of feeding tube placement: a diagnostic tool identifying gastrointestinal anomalies. Neonatal Netw. 2013; 32(2);89-94. doi: 10.1891/0730-0832.32.2.89.

25. ENA Clinical Practice Guideline Committee. Clinical Practice Guideline: Gastric Tube Placement Verification. J Emerg Nurs. 2019; 45(3); 306.e1-306.e19. doi: 10.1016/j.jen.2019.03.011.

26. Mordiffi SZ, Goh ML, Phua J, et al. Confirming nasogastric tube placement: Is the colorimeter as sensitive and specific as X-ray? A diagnostic accuracy study. International Journal of Nursing Studies. 2016; 61; 248-257. DOIه10.1016/j.jnurstu.2016.06.011

27. Ackerman $\mathrm{MH}$, Mick DJ. Technologic approaches to determining proper placement of enteral feeding tubes. AACN Adv Crit Care.2008; 17(3); 246-249. doi: 10.4037/15597768-2006-3002.

28. Bennetzen LV, Håkonsen SJ, Svenningsen H, et al. Diagnostic accuracy of methods to verify nasogastric tube position in mechanically ventilated adult patients: a systematic review. JBI Database System Rev Implement Rep. 2015; 13(1); 188-223. doi: 10.11124/jbisrir-2015-1609.

29. Lira D, Padole A, Kalra MK, et al. Tube potential and CT radiation dose optimization. AJR Am J Roentgenol. 2015; 204(1); W4-10. doi: 10.2214/AJR.14.13281.

30. Browne JE, Bruesewitz MR, Thomas V, et al . Procedure for optimal implementation of automatic tube potential selection in pediatric $\mathrm{CT}$ to reduce radiation dose and improve workflow. J Appl Clin Med Phys, 2021; 22(2);194-202. doi: 10.1002/acm2.13098.

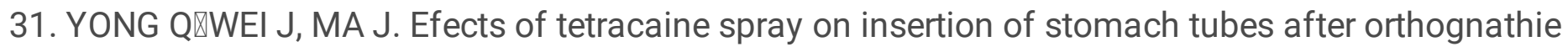

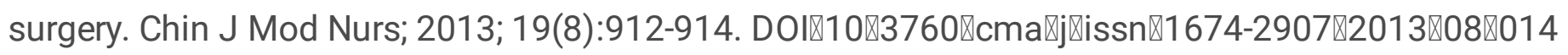

32. Yang Y, Du X, Tao XH. Application of Tetracaine Hydrochloride in Indwelling gastric Tube. Int J Nurs.

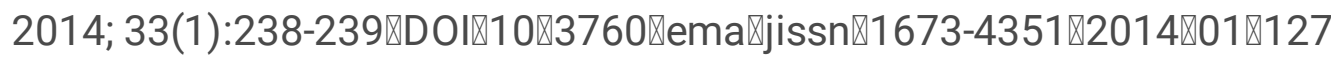

33. Okabe T, Goto G, Hori Y, et al. Gastric tube insertion under direct vision using the King Vision video laryngoscope『a randomized \prospective『clinical trial『BMC Anesthesiology. 2014; 14(82);1-5. doi: 10.1186/1471-2253-14-82.

34. Liu Wen-qin $\llbracket$ Shang Yi, an Hui-j iaa et al. Application of video laryngoscope in patients with gastric tube placement $\triangle a$ Meta-analysis. Journal of Lanzhou University(Medical Sciences). 2020;46(5);8-14.

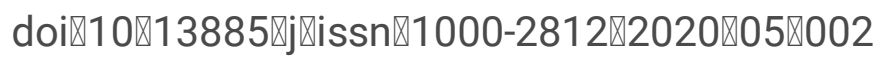

35. Kavakli AS, Kavrut Ozturk N, Karaveli A, et al. Comparison of different methods of nasogastric tube insertion in anesthetized and intubated patients $₫$ Brazilian Journal of Anesthesiology(English Edition). 2017;67(6);578-583. doi: 10.1016/j.bjan.2017.04.020.

36. Lee XL, Yeh LC, Jin YD, Chen CC, et al. Nasogastric tube placement with video-guided laryngoscope: A manikin simulator study. J Chin Med Assoc. 2017;80(8); 492-497. doi:

10.1016/j.jcma.2017.01.009.

\section{Figures}




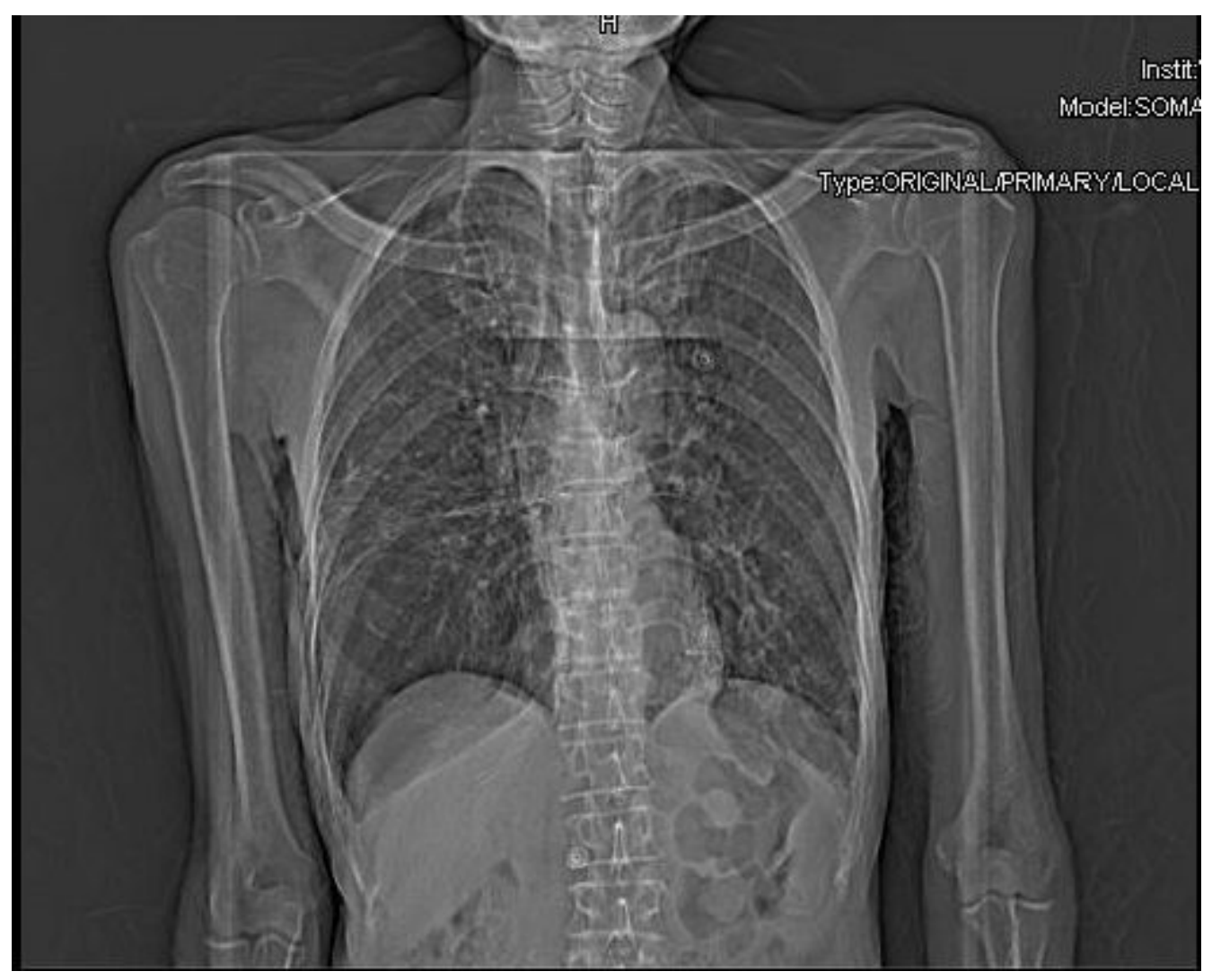

Figure 1

Chest X-ray at admission to ED (March 6, 2021). 


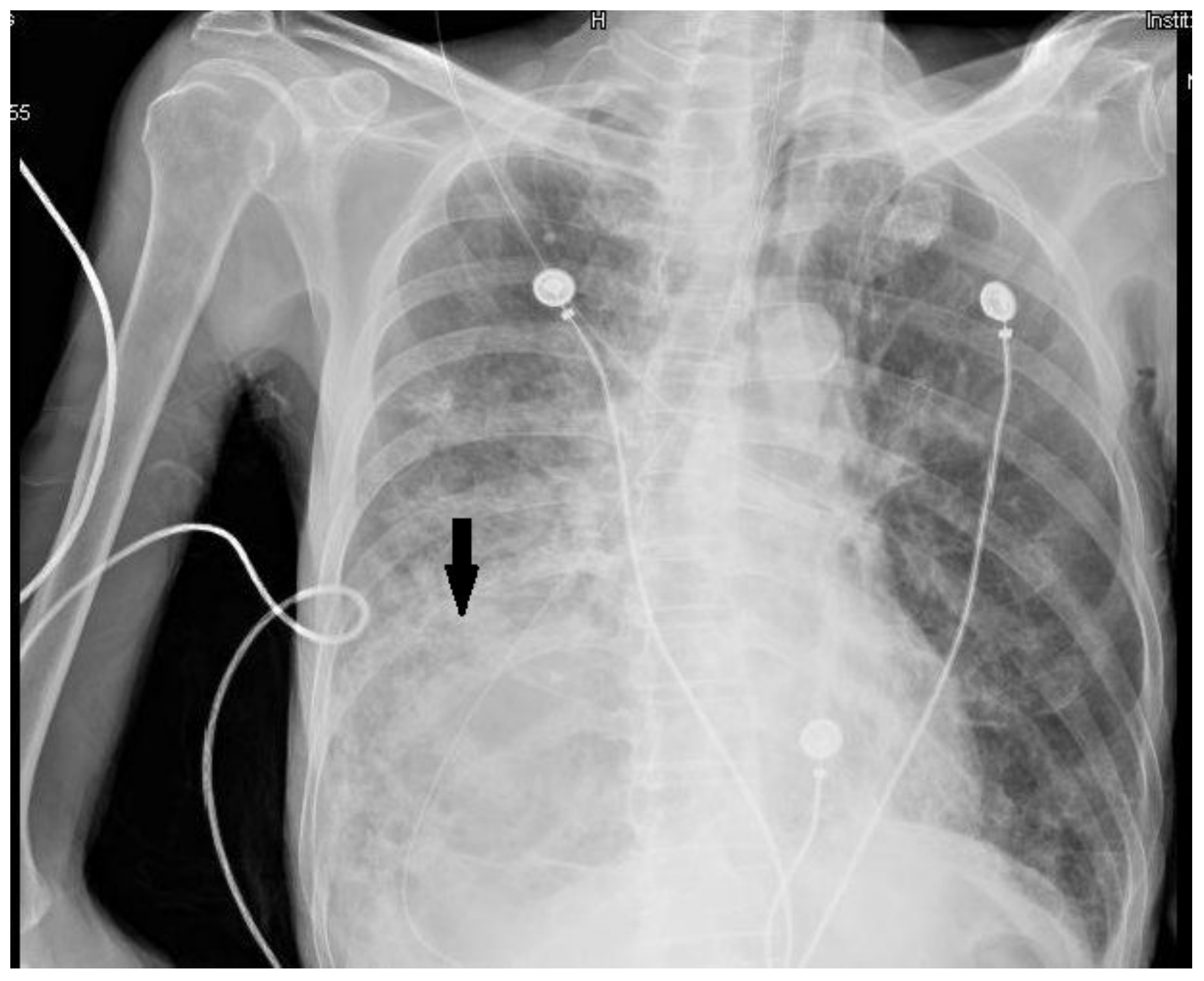

Figure 2

Chest X-ray reveals patchy, diffuse reticular-nodular opacities in bilateral lung fields, airspace consolidation with air bronchograms in the right lower zone. The black arrow pointed to the large highdensity shadow in the inferior lobe of right lung (March 15, 2021). 


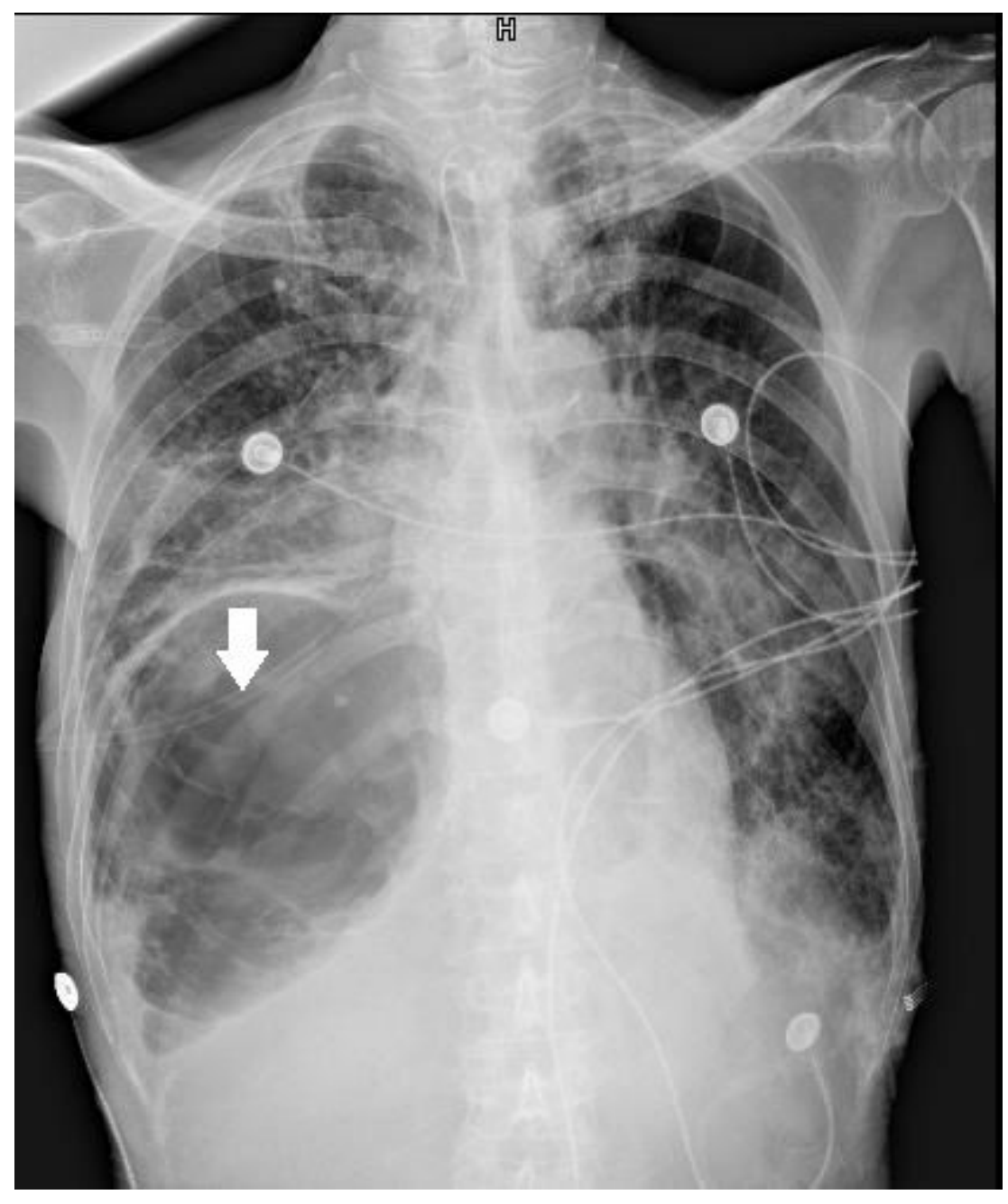

\section{Figure 3}

Chest X-ray revealed the large high-density shadow in the inferior lobe of right lung reduced compared with the radiographic image on March 15 (March 17, 2021). 